\title{
Robust Face Label Graph Matching for Movie Character Identification
}

\author{
Umesh Sawadekar \\ M. TechScholar \\ Dept. of Computer Science \\ Engineering, RGPV \\ Bhopal, M.P., State, India
}

\author{
Umesh Lilhore \\ Associate Prof. \\ Dept. of Computer Science \\ Engineering, RGPV \\ Bhopal, M.P., State, India
}

\author{
Sitendra Tamalkar \\ Associate Prof. \\ Dept. of Computer Science \\ Engineering, RGPV \\ Bhopal, M.P., State, India
}

\begin{abstract}
Being a successful application in the field of image analysis, face recognition is seeking most of the attention during the past few years. It is a difficult problem because of the huge variation in the appearance of each character and the weakness and ambiguity of available annotation. Robust Face Name Graph Matching for Movie Character Identification is used to detect the faces of the movie characters and the system designed is taking the least time to detect the face. Through the proposed system, we will be able to do it in a one minute process.
\end{abstract}

\section{Keywords}

Automatic Face Detection, Face Recognition,

Principal Component Analysis.

\section{INTRODUCTION}

Conventionally, the management of identity was almost satisfactorily achieved through connection of attributed identifiers with biometric identifiers which were attached in existing local social relations. As population is growing, communities have become more fleeting and individuals have started more traveling. Thus, the management of individuals needs a system of character management that will be more robust and supple. As, passwords and PINs are difficult to recall every time and can be stolen or predicted; tokens, keys, cards and other similar tools can be misplaced, replicated or forgotten, purloined; magnetic cards may become corrupted and unreadable. On the other side, biological traits can never be forged, forgotten, replicated or stolen.[1]

Biometric technologies have emerged as favorable tools to meet the difficulties in identification on the basis of belief that body doesn't lie and on the intense progress in a range of significant technologies. Biometric technologies identify on the basis of physiological features and behavioral traits. Face recognition offers many advantages over other biometric methods. All other technologies need some voluntary action from user side but face recognition can be done without any explicit action or participation by user.

\section{RELATED WORK}

Some of the related works about the automatic face identification and face expressions detection as follows:CsabaCzirjek [3] proposed to our approach the content structuring is to build an index based on the reappearance of the main characters within the content. For news programs, this can be used for temporal segmentation into individual news stories based on the fact that the anchorperson, the main character in this scenario signals the beginning of a news item. For movie content, this could provide enhanced random access browsing functionality to the end user. In this thesis we propose an approach to news story segmentation that uses low-level features and three different algorithms for temporal segmentation. We then extend this system to perform anchor-person detection using automatic face detection and clustering algorithms. An extensive manually marked up test set has been used to validate each component of our overall approach.

VinayBettadapura [4] proposed the paper of the automatic recognition of facial expressions and has been an active research topic since the early nineties. The paper presents a time-line view of the advances made in this field, the applications of automatic face expression recognizers, the characteristics of an ideal system, the databases that have been used and the advances made in terms of their standardization and a detailed summary of the state of threat. The paper also discusses facial parameterization using FACS Action Units (AUs) and MPEG-4 Facial Animation Parameters (FAPs) and the recent advances in face detection, tracking and feature extraction methods.

OgnjenArandjelovi'c and Andrew Zisserman [5] proposed that the objective of this work is to recognize all the frontal faces of a character in the closed world of a movie or situation comedy, given a small number of query faces. This is challenging because faces in a feature-length film are relatively uncontrolled with a wide variability of scale, pose, illumination, and expressions, and also may be partially occluded. They develop a recognition method based on a cascade of processing steps that normalize for the effects of the changing imaging environment.

Yi-Fan Zhang, ChangshengXu, Hanqing Lu and Yeh-Min Huang approach the paper for Identification of characters in films, although very intuitive to humans, still poses a significant challenge to computer methods. In this paper, we investigate the problem of identifying characters in feature length films using video and film script. The contributions of our work include a graph matching method, an effective measure of face track distance and the relationship between characters is mined using social network analysis. The proposed framework is able to create a new experience on character-centered film browsing.

Mengdi XU, xiaotong Yuan, JialieShen and ShuichengYan [6] proposed this work to recognize all the frontal faces of a character in the closed world of a movie or situation comedy, given a small number of query faces. This is challenging because faces in a feature-length film are relatively controlled 
with a wide variability of scale, pose, illumination, and expressions, and also may be partially occluded. We develop a recognition method based on a cascade of processing steps that normalize for the effects of the changing imaging environment.

Enrique G. Ortiz, Alan Wright, and Mubarak Shah ,they presents an end-to-end video face recognition system, addressing the difficult problem of identifying a video face track using a large dictionary of still face images of a few hundred people, while rejecting unknown individuals. A straight forward application of the popular $\ell 1$-minimization for face recognition on a frame-by-frame basis is prohibitively expensive, so we propose a novel algorithm

Mean Sequence SRC (MSSRC) that performs video face recognition using a joint optimization leveraging all of the available video data and the knowledge that the face track frames belong to the same individual. By adding a strict temporal constraint to the $\ell 1$-minimization that forces individual frames in a face track to all reconstruct a single identity, we show the optimization reduces to a single minimization over the mean of the face track.

\section{Problems with the existing system:}

- In the existing system, The faces of the movie characters are detected and the characters are recognized but a lot of time for detecting the faces in the windows processed.

- Due to the generation of noises during the tacking of faces and their clustering processes, performances are limited. Therefore, a lot of time is taken in processing and a lot of power is consumed.

\section{System Proposed:}

In this system, faces of movie characters are detected and minimum time is taken by the planned system to detect the face. In this system, we will be able to do it in one minute process.

The process of recognition of faces is of four interconnected steps. The procedure begins with detection of face which is followed by normalization. Then the features of the faces are extracted and finally recognition of face is done.

\section{Step 1: Detecting a face}

For detecting a face, the computer has to decide which pixels in the image are parts of the image and which are

Table 1

Tools used during the study

Tools

Visual Studio Used to design and run the system

By adding some extra features we can improvise the system. For this purpose, the following algorithms are used.

1. EGCM: This is known as Error Correcting Graph Matching Algorithm. It is used for detection of faces and to reduce the noises present in the complicated movie scenes. It defines various graph editing operations as per the noise analysis and then designs the edit cost function to improve the performance.

2. $P C A$ - Eigen Faces: This Principal Component Analysis technique converts the two dimensional images into a single dimensional vector. Every single component i.e. Eigenface has only one certain feature of the face. This feature may or may be present in the original image. Comparison of a probe image is done with the image in gallery by calculating the distance between their respective vectors. PCA is sensitive to the scale variations. In PCA, the gallery image must be similar to the probe image in terms of poses, illuminations and scales.

This is a difficult process for computer than humans especially when the background is filled with many objects.

Step 2: Normalization of faces

After the face has been detected, it needs to be normalized i.e. the main landmarks of a face must be positioned accurately and then the images for a little variation are corrected. These corrections are done on the basis of statistical interferences which may not be entirely precise.

\section{Step 3 and 4: Feature Extraction of faces and Facial Recognition}

Extracting the facial features is done by a mathematical demonstration which produces a biometric template. This biological reference is then stored in the database and forms the basis of facial recognition tasks. It is necessary for to take the maximum information to make the biometric template unique. It will help in a successful facial recognition.

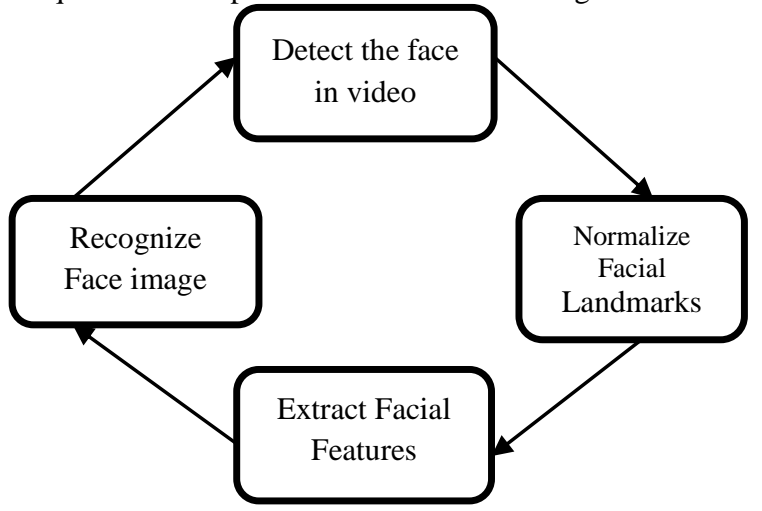

Figure 1: Steps in facial recognition process

$P C A$ - It was invented by Karl Pearson and nowadays it is mostly used as a tool in explanatory analysis of data for making predictive tools. It is the simplest of the true eigenvector-based multivariate analysis. Principal Component Analysis is used to reduce the dimension. PCA is going to find the most accurate data representation under face recognition i.e. the maximum variance in a lower dimension space.

PCA is a mathematical procedure that uses an orthogonal transformation to convert a set of values of possibly correlated $\mathrm{M}$ face images into a set of values of $\mathrm{K}$ uncorrelated variables called Eigenfaces. The number of Eigenfaces is always less than or equal to the number of face images. i.e. $\mathrm{K}<\mathrm{M}$. 
Principal Components shows the directions/features of the data and each proceeding component shows less directions and more noise. Therefore, only first few principal components, $\mathrm{K}$ are selected and the rest of the last components are discarded. Thus, each variable in the original dataset can be represented in terms of $\mathrm{K}$ principal components and reducing the data point this way reduces the number of values i.e. from $\mathrm{M}$ to $\mathrm{K}$ needed to recognize it and this makes the recognition process faster and freer of error caused by noise

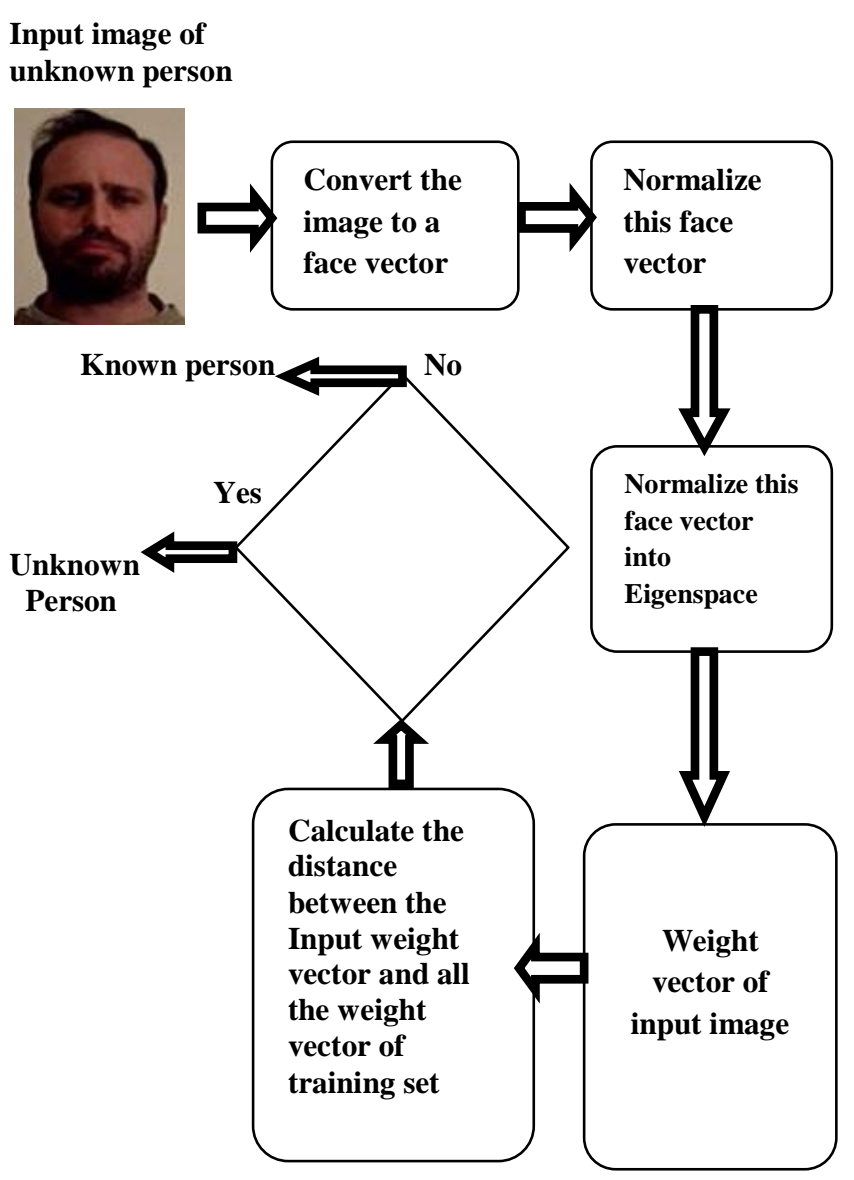

Fig 2. Flow chart of System

Step 1:

Create the training set

Step 2:

Convert face images in training set into face vectors

Step 3:

Normalize the face vectors

Step 4:

Reduce the dimensionality of the training set

Step 5:

Calculate the eigenvectors from covariance matrix.

Step 6:

Select $K$ best eigen faces, such that $K<M$ and represent the whole training set.

Step 7:

Convert lower dimensional $\mathrm{K}$ eigenvectors to original face dimensionality.

Step 8:

Represent each face image a linear combination of all $\mathrm{K}$ eigenvectors
Step 9:

For each image, calculate and store associates weight factors.

Step 10:

Calculate distance between input weight vector and all weight vectors of training set.

Then if the distance is less than the threshold, then the face is said to be known face otherwise the face is unknown

\section{SYSTEM ARCHITECTURE}

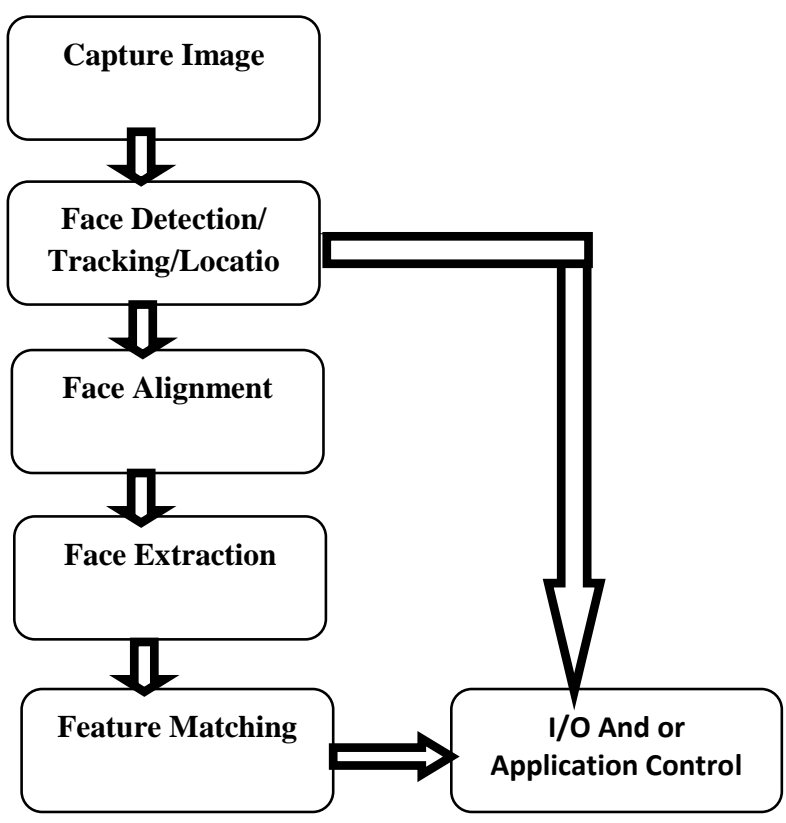

\section{CONCLUSION}

The main objective of the paper is to overcome the limitations of previous system by improving results for clustering and identifying the faces extracted from uncontrolled movie videos. Using sensitivity analysis, we have seen that such schemes have better robustness to the noises in constructing affinity graphs than the traditional methods. Any face recognition system could not provide $100 \%$ efficiency till now, but this system would provide efficiency up to $90 \%$ and thus error rate declines.

\section{FUTURE SCOPE}

The future enhancements of this system include that the facename matching technique should be developed in live video surveillance. Also, the system can be extended to detect faces in cases of external items like spectacles, helmet, cap etc. Further, the system can be extended to match even if the person is in case of age progression or disguise. Detecting the face of the person should be displayed with the details of the person in a small time interval.

\section{REFERENCES}

[1] Y. Zhang, C. Xu, H. Lu, and Y. Huang, "Character identification in feature-length films using global facename matching," IEEE Trans.Multimedia, vol. 11, no. 7, pp. 1276-1288, November 2009.

[2] J. Yang and A. Hauptmann, "Multiple instance learning for labeling faces in broadcasting news video," in ACM Int. Conf. Multimedia, 2005, pp. 31-40.

[3] CsabaCzirjek m. eng. in electronic engineering at dublin city university main character detection in news and movie content Copyright by CsabaCzirjek, 2005. 
[4] VinayBettadapura College of Computing, Georgia Instituteof Technology vinay@gatech.edu Face ExpressionRecognition and Analysis: The State of the Art

[5] OgnjenArandjelovi'c Engineering Department oa214@cam.ac.uk and Andrew Zisserman University ofOxford, UK, az@ robots.ox.ac.uk-“Automatic FaceRecognition for Film Character Retrieval in FeatureLengthFilms".
[6] Yi-Fan Zhang, Student Member, IEEE, ChangshengXu,Senior Member, IEEE, Hanqing $\mathrm{Lu}$, Senior Member, IEEE, and Yeh-Min Huang, Member, IEEE-“ CharacterIdentification in Feature-Length Films Using Global Face-Name Matching”- IEEE TRANSACTIONS ONMULTIMEDIA, VOL. 11, NO. 7 , NOVEMBER 2009. 